\title{
Complete revascularization via left snuffbox approach in a nonagenarian patient with acute myocardial infarction
}

\author{
Kirill Berezhnoi ${ }^{1,2}$, Leonid Kokov, ${ }^{2,3}$, Aleksandr Vanyukov ${ }^{1}$, Yongcheol Kim ${ }^{4}$ \\ ${ }^{1}$ Department of Interventional Cardiology, City Clinical Hospital No. 52, Moscow, Russian Federation \\ ${ }^{2}$ Department of Radiology, I.M Sechenov First Moscow State Medical University, Moscow, Russian Federation \\ ${ }^{3}$ Department of Interventional Cardiology, N.V. Sklifosovsky Institute, Moscow, Russian Federation \\ ${ }^{4}$ Department of Cardiology, Chonnam National University Hospital, Gwangju, Republic of Korea
}

A 94-year-old woman was admitted to the documented hospital with a 1-week history of intermittent angina. The electrocardiogram showed ST-segment depression in inferolateral leads and a high sensitivity troponin level was elevated. Echocardiography demonstrated hypokinesia of inferior, posterior and lateral walls. Urgent coronary angiography (CAG) was planned via the right radial approach after loading of acetylsalicylic acid $300 \mathrm{mg}$ and clopidogrel $300 \mathrm{mg}$, but the right radial artery was pulseless. Therefore, CAG was performed via the left distal radial approach in the area of the "anatomical snuffbox" (Fig. 1A, B, Suppl. Video 1). CAG demonstrated diffuse intermediate to severe stenosis in proximal to mid right coronary artery (RCA) (Fig. 1C, left) and severe stenosis in the circumflex artery (LCx) and obtuse marginal branch (Fig. 1D, left). Thus, complete revascularization was achieved with $4.0 \times 9 \mathrm{~mm}$ and $3.5 \times 38 \mathrm{~mm}$ zotarolimus-eluting stents (ZESs) in the RCA (Fig. 1C, right) and a $3.0 \times 24 \mathrm{~mm}$ ZES in the $\mathrm{LCx}$ (Fig. 1D, right). There was no bleeding complication of the puncture site with a compressive bandage with gauze for $3 \mathrm{~h}$ (Fig. 1E, F).

Regarding complete revascularization in patients with acute myocardial infarction, the feasibility of the distal radial artery approach, called snuffbox approach, has not been known well [Kim et al., Korean Circ J. 2018; 48: e118]. Furthermore, percutaneous coronary intervention (PCI) via the right conventional radial approach for very old patients is sometimes challenging due to a difficulty of catheter manipulation by severe subclavian tortuosity leading to crossover to femoral access, even though the transradial approach significantly reduces vascular complication in elderly patients. This case highlights the feasibility of mutivessel PCI via the left snuffbox approach in a very old patient with acute myocardial infarction.

Conflict of interest: None declared

Address for correspondence: Yongcheol Kim, MD, Department of Cardiology, Chonnam National University Hospital, 42 Jebong-ro, Donggu, Gwangju 61469, Republic of Korea, tel: 82-62-220-6246, fax: 82-62-223-3105, e-mail: Dr.YongcheolKim@gmail.com

Received: 22.07.2018 Accepted: 22.07.2018 


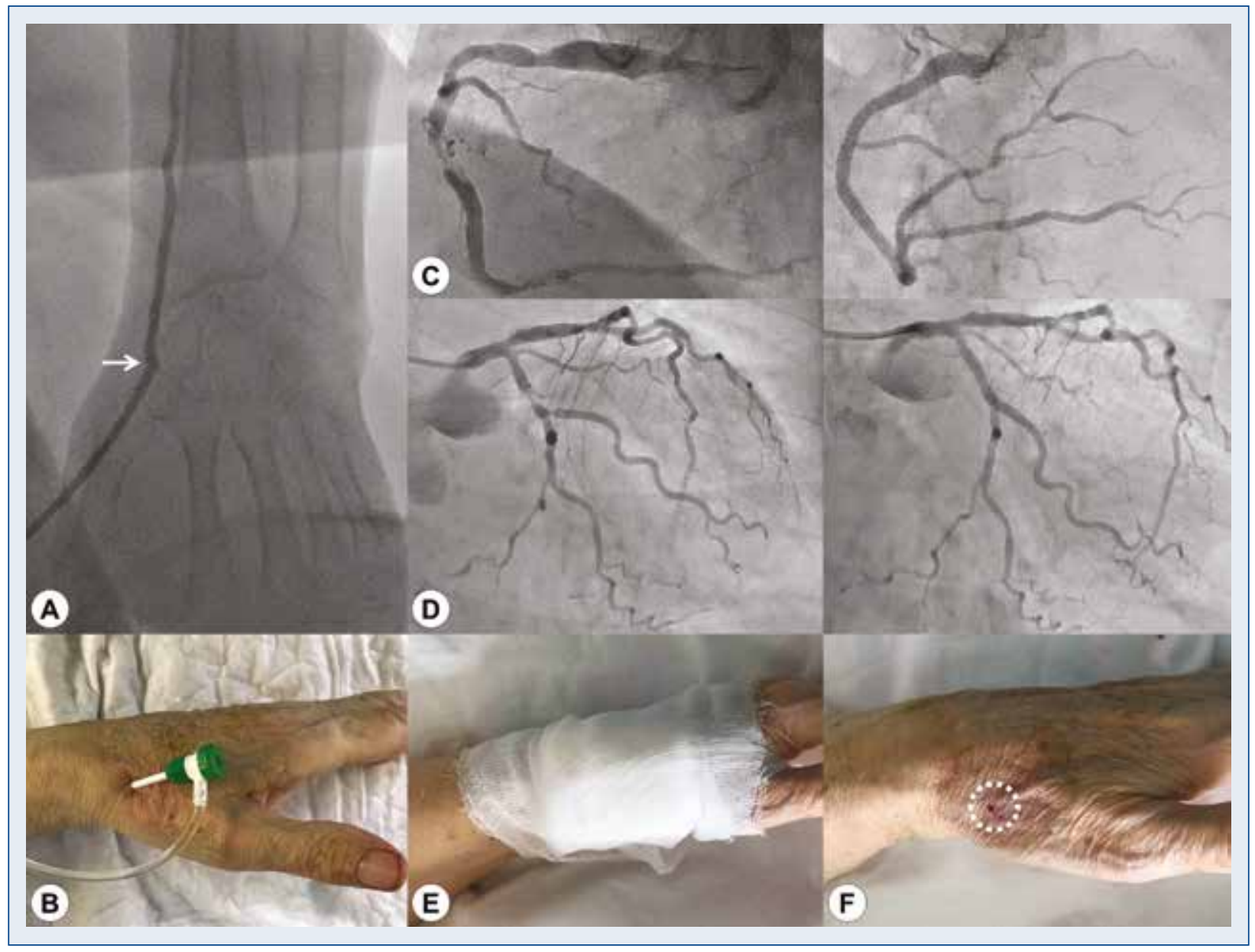

Figure 1. A. Peripheral angiography of left hand demonstrating successful puncture of the left distal radial artery (arrow: puncture site of left snuffbox approach); B. Inserted 6 Fr sheath via left snuffbox approach; C. Pre- (left) and post-interventional (right) coronary angiography in the right coronary artery; D. Pre- (left) and post-interventional (right) coronary angiography in the left circumflex artery; E. Hemostasis by manual compressive bandage with gauze; F. No vascular complication of puncture site the following day (white circle). 